\title{
Average Age of Information in Wireless Powered Relay Aided Communication Network
}

\author{
Yali Zheng, Jie Hu, Senior Member, IEEE, Kun Yang, Senior Member, IEEE
}

\begin{abstract}
In order to satisfy timeliness requirements arising from environmental sensing applications, age of information (AoI) was proposed to characterise the freshness of the received updates. In this paper, we consider a wireless powered relay aided communication network (WPRCN), in which a relay wirelessly powered by a hybrid-access point $(\mathrm{H}-\mathrm{AP})$ receives environmental monitoring information update from a sensor and forwards it to the H-AP. The long-term average AoI is studied since the decision correctness depends on the update timely uploaded by the relay. The wireless powered relay adopts either a decode-and-forward (DF) or an amplify and forward (AF) protocols, respectively, subject to the energy causality. We also consider the decoding cost of DF protocol owing to the relay's limited energy storage. Since the expression of the average AoI is non-elementary, we propose a Taylor approximation based algorithm to obtain its integral. We optimise the transmit power/the equivalent average power consumption of the relay for the sake of minimising the average AoI in the whole WPRCN with different forwarding protocols. Our simulation results demonstrate the accuracy of the theoretical analysis, while the average AoI of the WPRCN is optimised by our power allocation scheme at the relay. The proposed algorithm is verified to achieve great approximation effect.
\end{abstract}

Index Terms-Age of information (AoI), wireless power transfer (WPT), two-hop, relay, amplify and forward (AF), decode and forward (DF), decoding cost.

\section{INTRODUCTION}

Wireless power transfer (WPT) enabled by dedicated radio frequency $(\mathrm{RF})$ signals is capable of providing flexible, controllable and on-demand energy supply to massively deployed low-power Internet of Everything (IoE) devices, in order to support their sensing, computing and communication functions [1]. As a result, it enables so-called wireless powered communication networks (WPCNs) [2].

So far, tremendous efforts have been invested in the optimal design of WPCNs with different goals, such as throughput [3][6], energy efficiency [7], coverage probability [8], and diversity gain [9], etc. However, the timeliness issue incurred by the

Yali Zheng and Jie Hu are with the School of Information and Communication Engineering, University of Electronic Science and Technology of China, Chengdu 611731, China, and also with the Yangtze Delta Region Institute (Huzhou), University of Electronic Science and Technology of China, Huzhou 313001, P. R. China (email: yali_zheng@foxmail.com; hujie@uestc.edu.cn).

Kun Yang is with the School of Information and Communication Engineering, University of Electronic Science and Technology of China, Chengdu, 611731, China, and also with the School of Computer Science and Electronic Engineering, University of Essex, Colchester, CO4 3SQ, U.K. (email: kunyang@uestc.edu.cn).

The authors would like to thank the financial support of National Natural Science Foundation of China (NSFC), Grant No. U1705263, 61620106011, 62132004 and 61971102 .

Corresponding author: Jie Hu

Copyright (c) 2021 IEEE. Personal use of this material is permitted. However, permission to use this material for any other purposes must be obtained from the IEEE by sending a request to pubs-permissions@ieee.org. intermittent WPT in environment monitoring applications is ignored. In these special applications, only timely updates can reflect genuine status of dynamic environments, while outdated update is meaningless and even misleading. Moreover, in some time-sensitive applications, such as monitoring of a cyberphysical system [10], the traditional communication delay referring to the time elapsed from the update generation to its reception, which is incurred by processing, queuing, sending and propagating, is not a sufficient measurement of timeliness [11]. When a batteryless device cannot upload timely updates due to insufficient energy supply, the receiver cannot obtain the current state of the device neither. Therefore, age of information (AoI) is proposed as a new measurement to characterise the freshness of uploaded updates [12], [13]. Specifically, AoI is defined as the time elapsed since the generation of the freshest update.

In order to address timeliness challenges in energy harvesting based communication systems with intermittent energy supply, AoI has attracted many endeavours invested in it [11], [14]-[32]. Some recent works maintained update freshness in one-hop transmission [14]-[22]. Specifically, by considering a finite battery, the long-term average AoI was minimised in [14]. Moreover, Bacinoglu et al. [15] attained the lowest time-average "age penalty", which measured the timeliness of uploaded updates with a non-decreasing penalty function. The average peak AoI was obtained by considering a non-linear penalty function in [16]. Wu et al. [17] further minimised the average AoI with causal energy constrains by considering different battery size. Krikidis [18] minimised the average AoI by optimising the capacity of energy storage unit. Given a fixed channel power gain, a resource allocation algorithm to minimise the average AoI was proposed in an energy harvesting based wireless sensor network (WSN) [19]. Furthermore, there are several related works focusing on both access control [20] [21] and power control [22].

The AoI performance was also investigated in WPCNs [23][26] with multiple devices. There are several works focusing on AoI minimisation for WPCNs by exploiting either conventional resource allocation [23] or emerging deep reinforcement learning (DRL) [24]. In [25], the energy outage probability was minimised, while maintaining the AoI below a certain level in a data generation control system with the support of energy harvesting. The proposed optimal policy was capable of achieving a comparable energy outage probability to the conventional energy-oriented scheme. Furthermore, Zheng et al. [26] minimised the AoI in a WPCN with the assistance of multiple selfish wireless nodes, which requires instantaneous feedback of the channel state information (CSI).

Moreover, AoI was also investigated in two-way data ex- 
changing systems enabled by WPT [11] [27] and in twohop energy harvesting aided communication systems [28][32]. Specifically, $\mathrm{Hu}$ et al. [11] analysed and minimised the average weighted-sum-AoI between downlink and uplink communications by optimising the power-splitting ratio and the weighting coefficient. Dong et al. [27] further investigated the trade-off between the average downlink and uplink AoIs in a fog computing system with a single wireless powered mobile device. Moreover, Arafa et al. [28] studied the AoI of an energy harvesting aided two-hop communication system, in which a single relay working in a half-duplex (HD) mode was powered by energy harvested from ambient environment. This work was further extended to an AoI minimisation problem by satisfying energy causality and service time constraints [29]. Zhou et al. [30] optimised the freshness of real-time data for energy harvesting based two-hop systems with multiple devices subject to energy constraints. Furthermore, the peak AoI was investigated by analysing the capacity of relay's capacitor in a wireless powered cooperative network, where a relay powered by a power station adopts a decode-andforward (DF) and an amplify-and-forward (AF) protocols, respectively [31]. However, the decoding cost of the relay with a DF protocol was not be considered in detail. Moreover, the average AoI in simultaneous wireless information and power transfer (SWIPT) enabled cooperative communication system was minimised in case of a fixed information rate [32].

Unfortunately, the existing works [11], [19], [26]-[32] have the following drawbacks:

- The average AoI performance of a practical update forwarding protocol, such as an AF and a DF, was not analysed and evaluated, when an H-AP is invoked for both downlink WPT and uplink update reception.

- Their quantification of the energy consumption was simple. For instance, they only assumed that an information update consumes a single energy packet harvested from ambient environment.

- They did not consider the energy consumption for the update decoding, which is essential for a wireless powered relay with scarcity energy storage.

- Numerical calculations on the average AoI were obtained by block-integral with a high complexity, which contradicts the original purpose of obtaining analytical results for achieving fast-speed calculations.

In order to overcome the aforementioned drawbacks, our novel contributions are summarised as below:

- We study a wireless powered relay aided communication network (WPRCN) by focusing on its long-term average AoI performance, where either a DF or an AF protocol is invoked for relaying timely update from the sensor to the $H$-AP. The relay operates in a frequency division duplex (FDD) mode. Therefore, it can simultaneously harvest energy from the downlink transmission of the H-AP and receive updates from the uplink transmission of the sensor.

- We consider the energy consumption of the relay for the update decoding, when the DF protocol is adopted, owing to the limited energy supply. The WPRCN without considering the decoding cost is regarded as a benchmark to demonstrate its impact on the average AoI.

- By considering the energy causality at the relay and the successful transmission probability at the $H$-AP, the average AoI is quantified by conceiving a discrete-time model and it is obtained in closed-form, which depends on the transmit power/the equivalent average power consumption of the relay. The successful transmission probability is transformed into an elementary function via an error control based Taylor approximation method, which can effectively avoid the traditional block integral.

- We propose an offline design to minimise the average AoI per transmission block by optimising the transmit power/the equivalent average power consumption of the relay. Our design does not need any instantaneous feedback.

- Our simulation results demonstrate the accuracy of our theoretical analysis, and the optimality of our averageAoI-minimisation-oriented design. The proposed error control based Taylor approximation method is verified to have a high accuracy. According to the average AoI analysis, capacitors with optimal capacity $B^{*}$ (or those with a capacity closest to $B^{*}$ according to the practical supply of capacitors) should be selected in the practical implementation. Moreover, in the case of sufficient energy supply, the AoI performance of the relay with a practical $D F$ protocol outperforms that with an AF protocol, while the latter may have a better performance in the case of a low outage-threshold.

The rest of this paper is organised as follows: Our system model is described in Section II, which is followed by the problem formulation in Section III. The optimisation problems for the WPRCN adopting the DF and AF protocols are solved in Section IV and $\mathrm{V}$, respectively, which are demonstrated by the numerical results in Section VI. This paper is finally concluded in Section VII.

\section{System ModeL}

\section{A. Network Model}

The model of the WPRCN is illustrated in Fig.1, which consists of a single hybrid-access point (H-AP), a relay and a sensor ${ }^{1}$. In particular, we consider a wireless environment without a direct link between the H-AP and the sensor. This is in line with a range of practical scenarios with numerous blockages, such as forest, mountain areas and etc. The H-AP equipped with a single transmit and a single receive antenna has the following pair of functions:

- The H-AP transmits dedicated RF signals in the downlink for providing wireless power to the batteryless relay.

\footnotetext{
${ }^{1}$ We do not consider two-hops WPT in our system. In the path-loss model, every hop may experience a $30 \mathrm{~dB}$ loss at least, which is the value at the reference distance $1 \mathrm{~m} \mathrm{[4].} \mathrm{The} \mathrm{adverse} \mathrm{effect} \mathrm{of} \mathrm{the} \mathrm{serious} \mathrm{path-loss}$ is also demonstrated by our experimental prototype [33]. Therefore, we have to suffer from $60 \mathrm{~dB}$ loss at least in two-hop WPT. Moreover, the recharging time of both the sensor and the relay greatly increases the AoI, which may result in the out-of-date or even invalid updates in the scenario with high requirements for the information timeliness. Hence, the WPT in more than one-hop transmissions has difficulty to be applied in practical implementations. Moreover, state of the art battery technique may also support sensors to work for a relatively long period [34].
} 

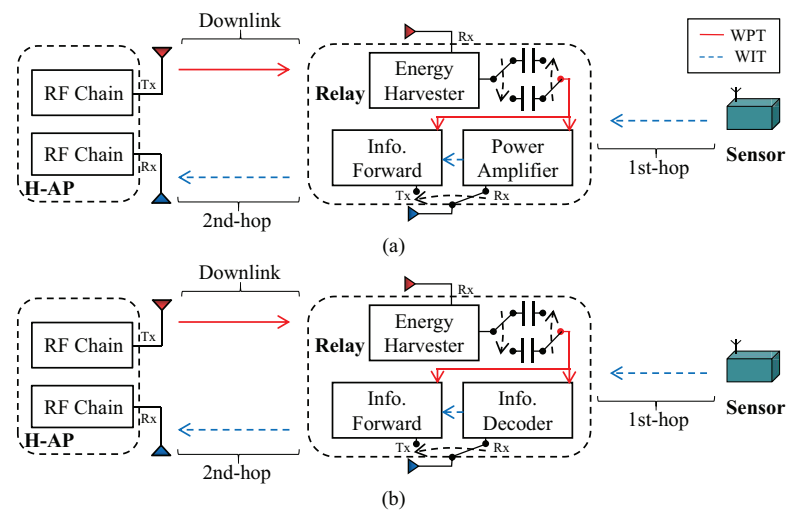

Fig. 1. The system model of WPRCNs with (a) an AF protocol and (b) a DF protocol.

- The H-AP operates in a FDD mode in order to simultaneously receive the uploaded updates from the sensor and to transfer WPT in the donwlink. Note that our system is also available for full-duplex aided systems with perfect self-interference cancellation.

The relay equipped with a pair of antennas ${ }^{2}$ has the following functions:

- The relay harvests RF energy from the downlink WPT of the H-AP via a dedicated antenna.

- The relay also operates in a FDD mode. Therefore, it can simultaneously carry out the updates uploading and the energy harvesting.

- The relay is equipped with two capacitors since the capacitor cannot charge and discharge at the same time. Each of them has a total energy capacity. Therefore, at any moment, there is always a capacitor being recharged, while the other is being discharged for update decoding and forwarding [35].

- We consider that the relay adopts either the AF or the DF protocols, respectively. As demonstrated in Fig. 1 (a), the relay with the AF protocol forwards the updates by directly amplifying the received uplink signal via its power amplifier, while it decodes and forwards the received update by adopting the DF protocol, as shown in Fig. 1 (b). Its update decoding and forwarding are both powered by the energy harvested from the downlink WPT of the H-AP. It only forwards the update to the H-AP, when one of its capacitors is fully charged.

The sensor is equipped with a single antenna for uploading the fresh update to the relay. A "zero-gap" principle is followed by the sensor, which means that the sensor immediately generates a fresh update as the previous one is received by the relay.

Both the H-AP and the relay adopt an automatic repeat request (ARQ) mechanism. Specifically, we consider that an

\footnotetext{
${ }^{2}$ The relay in the WPRCN can be readily extended to equip multiple antennas. In this case, the average AoI can be generally formulated similar to the case of the relay with a pair of antennas. However, the formulation of the average recharging time and the successful transmission probability may be changed owing to the distribution of the channel power gain, which may further have an impact on the solution to the optimisation problem. The details will be considered in our future work.
}

update can be successfully transmitted to the H-AP when the attainable spectral efficiency is higher than a predefined threshold, namely $\log (1+S N R) \geq r$. Otherwise, the update is retransmitted by the sensor and the relay.

All wireless channels experience uncorrelated block Rayleigh-fading [36]. A discrete-time model is adopted, where the $k$-th transmission block is defined as a period between the epoch $k$ and the epoch $(k+1)$. Hence, the normalised fading coefficient of the downlink channel from the H-AP to the relay during the $k$-th transmission block is represented by $h_{d k}$, while the normalised fading coefficients of the 1st- and 2nd-hop uplink channels are denoted as $h_{u 1 k}$ and $h_{u 2 k}$. Their conjugate transposes are expressed as $h_{d k}^{\dagger}, h_{u 1 k}^{\dagger}$ and $h_{u 2 k}^{\dagger}$, respectively. Furthermore, the large-scale path-loss in the downlink channel, and those in the 1st- and 2nd-hop uplink channels are denoted as $\Omega_{d}, \Omega_{u 1}$ and $\Omega_{u 2}$, respectively.

\section{B. Downlink WPT}

Without loss of generality, the H-AP is assumed to transmit a complex symbol $x_{d}$ for the downlink WPT, satisfying $\mathbb{E}\left[x_{d} x_{d}^{\dagger}\right]=1$, where $x_{d}^{\dagger}$ is the conjugate value of $x_{d}$. The corresponding downlink WPT signal $y_{d k}$ received by the relay during the $k$-th downlink transmission block is formulated as $y_{d k}=\sqrt{P_{t} \Omega_{d}^{-1}} h_{d k} x_{d}+n_{0}$, where $P_{t}$ is the transmit power of the H-AP and $n_{0}$ is the Gaussian distributed noise having a zero mean and a variance of $\sigma_{0}^{2}$.

The linear energy harvesting model is considered in the paper since it is sufficient for providing useful lower bounds of AoI for the WPRCN [18]. Therefore, the total energy harvested by the relay during the $k$-th transmission block is formulated as

$$
E_{d k}=\zeta P_{t} h_{d k} h_{d k}^{\dagger} \Omega_{d}^{-1} T_{b}
$$

where $\zeta(0<\zeta<1)$ is energy conversion efficiency and $T_{b}$ is the duration of a single transmission block. The noise power is neglected due to its negligibly small power.

Hence, the total energy $E_{k}$ stored in the capacitor with a capacity of $B$ after the $k$-th transmission block is expressed as

$$
E_{k}=\min \left\{\mathbb{1}_{E_{k-1}<B} \cdot E_{k-1}+E_{d k}, B\right\},
$$

where $\mathbb{1}_{\alpha}=1$ if the boolean variable $\alpha$ is true and $\mathbb{1}_{\alpha}=0$, otherwise. Note that the relay can decode and forward a new update only when the total energy $E_{k}$ stored in the capacitor satisfies $E_{k}=B$.

\section{Two-Hop Uplink Transmission}

The uplink update uploading has to be completed via twohop transmissions, namely the 1st-hop from the sensor to the relay and the 2nd-hop from the relay to the H-AP.

In the 1st-hop, the sensor transmits its update with a power of $P_{s}$. The WIT signal received by the relay's antenna during the $k$-th transmission block is obtained as $y_{u 1 k}=$ $\sqrt{P_{s} \Omega_{u 1}^{-1}} h_{u 1 k} x_{u k}+z_{0}$, where $x_{u k}$ satisfying $\mathbb{E}\left[x_{u k} x_{u k}^{\dagger}\right]=1$ is the modulated complex signal uploaded by the sensor and $z_{0}$ represents the Gaussian distributed noise received by relay's 
antenna, which has a zero mean and a variance of $\sigma_{0}^{2}$. Hence, the signal power received by the relay is expressed as

$$
P_{u 1 k}=P_{s} \Omega_{u 1}^{-1} h_{u 1 k} h_{u 1 k}^{\dagger}+\sigma_{0}^{2} .
$$

As a result, the received throughput at the relay during the transmission block $k$ is formulated as

$$
R_{u 1 k}=W T_{b} \log (1+\underbrace{\frac{P_{s} \Omega_{u 1}^{-1} h_{u 1 k} h_{u 1 k}^{\dagger}}{\sigma_{0}^{2}}}_{\text {Received SNR } S_{u 1 k}}),
$$

where $W$ represents the bandwidth of the channel.

In the 2nd-hop, the relay forwards its received update from the sensor to the H-AP by exploiting the energy harvested. The $\mathrm{DF}$ and the AF protocols are adopted by the relay, respectively. The 2nd-hop uplink transmission performance is analysed in the following three cases.

1) The WPRCN with an ideal DF protocol: With an ideal DF protocol, the relay does not consume any energy for the update decoding, which is in line with the most of existing literature [37], [38].

The signal and the corresponding power received by the $\mathrm{H}$-AP are expressed as

$$
\begin{gathered}
y_{i d f, k}=\sqrt{P_{r} \Omega_{u 2}^{-1}} h_{u 2 k} x_{u k}+n_{0}, \\
P_{i d f, k}=P_{r} \Omega_{u 2}^{-1} h_{u 2 k} h_{u 2 k}^{\dagger}+\sigma_{0}^{2},
\end{gathered}
$$

where we define $P_{r} \triangleq B / T_{b}$. Since the relay consumes all the energy stored in one of its capacitors for forwarding the update to the H-AP during a transmission block, $P_{r}$ is the transmit power of the relay with an ideal DF protocol. Therefore, the received $\mathrm{SNR}$ at the $\mathrm{H}-\mathrm{AP}$ is formulated as

$$
S_{i d f, k}=\frac{P_{r} \Omega_{u 2}^{-1} h_{u 2 k} h_{u 2 k}^{\dagger}}{\sigma_{0}^{2}} .
$$

2) The WPRCN with a practical DF protocol: With a practical DF protocol, the relay consumes its energy harvested for the successful update decoding. The update decoding power is obtained as

$$
P_{d c, k}=a\left(2^{R_{u 1 k} / T_{b}}-1\right),
$$

where $a$ is decoding cost factor [39] and $R_{u 1 k} / T_{b}$ is the information update transmission rate towards the relay during the transmission block $k$. Therefore, the total energy consumed for the update decoding during the $k$-th transmission block is expressed as $E_{d c, k}=P_{d c, k} T_{b}$. The signal and the corresponding power received by the H-AP are expressed as

$$
\begin{gathered}
y_{p d f, k}=\sqrt{\left(P_{r}-P_{d c, k}\right) \Omega_{u 2}^{-1}} h_{u 2 k} x_{u k}+n_{0}, \\
P_{p d f, k}=\left(P_{r}-P_{d c, k}\right) \Omega_{u 2}^{-1} h_{u 2 k} h_{u 2 k}^{\dagger}+\sigma_{0}^{2},
\end{gathered}
$$

where $\left(P_{r}-P_{d c, k}\right)$ is the transmit power of the relay. Note that $P_{r}$ denotes the equivalent average power consumption of the relay with a practical DF protocol. Therefore, the attainable uplink SNR at the H-AP is formulated as

$$
S_{p d f, k}=\frac{\left(P_{r}-P_{d c, k}\right) \Omega_{u 2}^{-1} h_{u 2 k} h_{u 2 k}^{\dagger}}{\sigma_{0}^{2}} .
$$

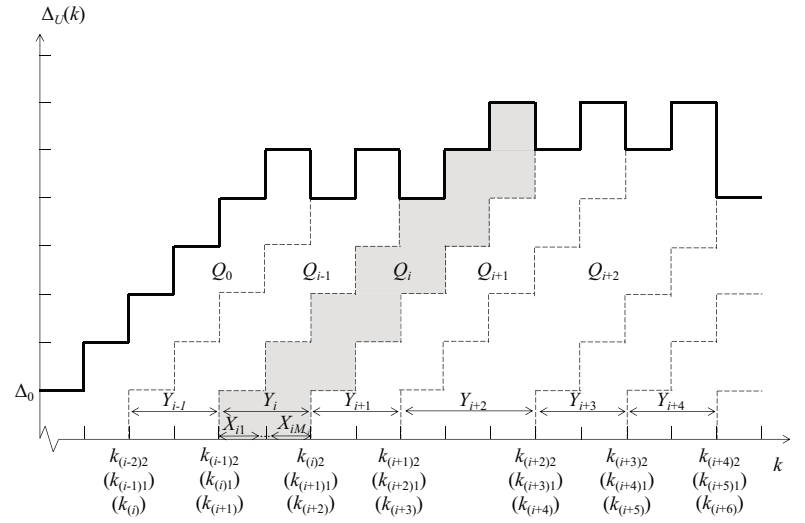

Fig. 2. Sample path of the uplink AoI $\Delta_{U}(k)$ (the upper envelop in bold).

3) The WPRCN with an AF Protocol: When the AF protocol is adopted, the signal received by the $\mathrm{H}-\mathrm{AP}$ is formulated as $y_{a f, k}=\sqrt{\Omega_{u 2}^{-1}} h_{u 2 k} \beta y_{u 1 k}+n_{0}$, where $\beta$ denotes the amplification factor of the relay. According to Eq. (3), $\beta$ can be expressed as

$$
\beta=\sqrt{\frac{P_{r}}{P_{u 1 k}}}=\sqrt{\frac{P_{r}}{P_{s} h_{u 1 k} h_{u 1 k}^{\dagger} \Omega_{u 1}^{-1}+\sigma_{0}^{2}}} .
$$

Therefore, the signal power received by the H-AP is expressed as

$$
\begin{aligned}
P_{a f, k} & =P_{s} \Omega_{u 1}^{-1} \Omega_{u 2}^{-1} h_{u 1 k} h_{u 1 k}^{\dagger} h_{u 2 k} h_{u 2 k}^{\dagger} \beta^{2}+\sigma_{0}^{2}\left(1+\Omega_{u 2}^{-1} h_{u 2 k} h_{u 2 k}^{\dagger} \beta^{2}\right), \\
& =\Omega_{u 2}^{-1} h_{u 2 k} h_{u 2 k}^{\dagger} P_{r}+\sigma_{0}^{2} .
\end{aligned}
$$

Hence, the received SNR at the H-AP is formulated as

$$
\begin{aligned}
S_{a f, k} & =\frac{P_{s} \Omega_{u 1}^{-1} \Omega_{u 2}^{-1} h_{u 1 k} h_{u 1 k}^{\dagger} h_{u 2 k} h_{u 2 k}^{\dagger} \beta^{2}}{\sigma_{0}^{2}\left(1+\Omega_{u 2}^{-1} h_{u 2 k} h_{u 2 k}^{\dagger} \beta^{2}\right)}, \\
& =\frac{P_{s} \Omega_{u 1}^{-1} \Omega_{u 2}^{-1} h_{u 1 k} h_{u 1 k}^{\dagger} h_{u 2 k} h_{u 2 k}^{\dagger} P_{r}}{\sigma_{0}^{2}\left(P_{s} h_{u 1 k} h_{u 1 k}^{\dagger} \Omega_{u 1}^{-1}+P_{r} h_{u 2 k} h_{u 2 k}^{\dagger} \Omega_{u 2}^{-1}+\sigma_{0}^{2}\right)}, \\
& \approx \frac{P_{s} \Omega_{u 1}^{-1} \Omega_{u 2}^{-1} h_{u 1 k} h_{u 1 k}^{\dagger} h_{u 2 k} h_{u 2 k}^{\dagger} P_{r}}{\sigma_{0}^{2}\left(P_{s} h_{u 1 k} h_{u 1 k}^{\dagger} \Omega_{u 1}^{-1}+P_{r} h_{u 2 k} h_{u 2 k}^{\dagger} \Omega_{u 2}^{-1}\right)} .
\end{aligned}
$$

Finally, the attainable uplink throughput at the H-AP in the above three cases during the transmission block $k$ may be formulated as

$$
R_{u 2 k}=W T_{b} \log \left(1+S_{k}\right),
$$

where $S_{k}=\left\{S_{i d f, k}, S_{p d f, k}, S_{a f, k}\right\}$.

\section{Problem Formulation}

AoI is defined as the time elapsed since the generation of a fresh update. In a discrete-time model, we assume that the AoI does not change within a single transmission block. Therefore, the AoI $\Delta_{U}(k)$ is the difference between the current epoch $k$ and the generation epoch $U_{U}(k)$, which is expressed as

$$
\Delta_{U}(k)=k-U_{U}(k) .
$$

Note that $U_{U}(k)$ is also the epoch when the previous update is successfully uploaded. 
Fig. 2 depicts a sample path of the AoI $\Delta_{U}(k)$ in the twohop uplink transmission, where $k_{(i)}$ denotes the epoch that the sensor generates the $i$-th update, while $k_{(i) 1}$ represents the epoch of the $i$-th update's 1st-hop transmission at the first time and $k_{(i) 2}$ is the epoch of the $i$-th update's 2nd-hop successful transmission. Note that when the $i$-th update is received by the relay via the 1st-hop transmission, the sensor immediately generates the next update at $k_{(i+1)}$ owing to the "zero-gap" principle. Therefore, $k_{(i) 1}$ and $k_{(i+1)}$ coincide with each other, as shown in Fig. 2. Since the relay receives the $i$-th update immediately, when the $(i-1)$-th update is successfully received by the H-AP, $k_{(i) 1}$ is equal to $k_{(i-1) 2}$. Therefore, $k_{(i-1) 2}, k_{(i) 1}$ and $k_{(i+1)}$ represent the same epoch.

Moreover, since the ARQ mechanism is adopted by both the $\mathrm{H}-\mathrm{AP}$ and the relay, the sensor may keep uploading the update to the H-AP block by block until the instantaneous spectral efficiency is higher than a threshold, i.e. $R_{\text {uik }} /\left(W T_{b}\right) \geq r$ for $i=1,2$, according to Eqs. (4) and (15). Obviously, a higher threshold $r$ results in an increasing AoI, since it requires more retransmission attempts before a successful transmission takes place. Hence, as shown in Fig. 2, $Y_{i}=k_{(i) 2}-k_{(i) 1}$ denotes the duration, when the $i$-th update can be successfully transmitted to the H-AP. Since the relay uploads the update only when one of its capacitors becomes fully charged, it satisfies $Y_{i}=$ $\sum_{j=1}^{M_{i}} X_{i j}$, where $X_{i j}$ represents the $j$-th complete recharging time during the $i$-th update's 2nd-hop transmissions, while $M_{i}$ is a random number of transmission blocks required for the relay to successfully deliver the update to the H-AP.

\section{A. Successful Transmission Probability}

In the 2nd-hop transmission, by considering $h_{d k} h_{d k}^{\dagger}$ for $k=$ $1, \cdots, K$ as independent and identically distributed exponential random variables, the probability mass function (PMF) of the number of transmission blocks $K$ being required for fully recharging one of the capacitors at the relay is obtained as [18]

$$
\Pi(\phi, K)=\frac{1}{(K-1) !}(\lambda \phi)^{K-1} \exp (-\lambda \phi),
$$

where $\phi=\frac{P_{r}}{\zeta P_{t} \Omega_{d}^{-1}}$. Then the first-order and the second-order moments for the recharging time $X$ of the relay are given by [18]

$$
\begin{aligned}
& \mathrm{E}[X]=1+\theta, \\
& \mathrm{E}\left[X^{2}\right]=1+3 \theta+\theta^{2},
\end{aligned}
$$

where $\theta=\frac{\lambda P_{r}}{\zeta P_{t} \Omega^{-1}}$. Similarly, those for a complete uploading time $Y$ of the relay are obtained as

$$
\begin{aligned}
& \mathrm{E}[Y]=\frac{1+\theta}{\pi}, \\
& \mathrm{E}\left[Y^{2}\right]=\frac{1+3 \theta+\theta^{2}}{\pi}+\frac{2\left(1+\theta^{2}\right)(1-\pi)}{\pi^{2}},
\end{aligned}
$$

where $\pi$ is the joint successful transmission probability in both the 1st- and 2nd-hop. This probability can be formulated as

$$
" \pi=\mathcal{P}\left\{\left(\log \left(1+S_{u 1 k_{1}}\right) \geq r\right) \cap\left(\log \left(1+S_{k_{2}}\right) \geq r\right)\right\},
$$

where $S_{k_{2}}=\left\{S_{i d f, k_{2}}, S_{p d f, k_{2}}, S_{a f, k_{2}}\right\}$. Note that $k$ in $S_{u 1 k}$ of Eq. (4) and that in $S_{k}$ of Eq. (15) are substituted by $k_{1}$ and $k_{2}$ in Eq. (22), respectively. Therefore, $S_{u 1 k_{1}}$ refers to the SNR of the received updates at the relay during the 1st-hop transmissions, while $S_{k_{2}}$ refers to the SNR of the updates received by the H-AP during the 2nd-hop transmissions. Since $\pi$ varies with different forwarding protocols, it will be formulated for three cases in Sections IV and V, respectively.

\section{B. AoI Quantification}

During the total transmission blocks $K \rightarrow \infty$, we suppose that the H-AP receives $N$ updates. Hence, the uplink transmission rate $v$ can be expressed as

$$
v=\lim _{K \rightarrow \infty} \frac{N}{K}=\frac{1}{\mathrm{E}[Y]},
$$

Furthermore, the average uplink AoI $\bar{\Delta}_{U}$ is expressed as

$$
\begin{aligned}
\bar{\Delta}_{U} & =\lim _{K \rightarrow \infty} \frac{1}{K} \sum_{k=1}^{K} \Delta_{U}(k), \\
& =\lim _{K \rightarrow \infty} \frac{1}{K}\left(\sum_{i=0}^{N-2} Q_{i}+\frac{1}{2} S_{N}\left(S_{N}+1\right)\right), \\
& \approx \lim _{K \rightarrow \infty} \frac{N-1}{K} \cdot \frac{1}{N-1} \sum_{i=0}^{N-2} Q_{i}, \\
& =v \cdot \mathrm{E}[Q],
\end{aligned}
$$

where $S_{N}=Y_{N-1}+Y_{N}$ is the service time of the $N$-th update. Moreover, we define $Q_{i}$ as the area under the curve $\Delta_{U}(k)$ in Fig. 2, which is formulated as

$$
\begin{aligned}
Q_{i}= & \frac{1}{2}\left(S_{i+1}+Y_{i+2}+1\right)\left(S_{i+1}+Y_{i+2}\right)-\frac{1}{2} S_{i+2}\left(S_{i+2}+1\right), \\
= & \frac{1}{2}\left(Y_{i}+Y_{i+1}+Y_{i+2}+1\right)\left(Y_{i}+Y_{i+1}+Y_{i+2}\right) \\
& -\frac{1}{2}\left(Y_{i+1}+Y_{i+2}\right)\left(Y_{i+1}+Y_{i+2}+1\right), \\
= & \frac{1}{2}\left(Y_{i}^{2}+2 Y_{i} Y_{i+1}+2 Y_{i} Y_{i+2}+Y_{i}\right) .
\end{aligned}
$$

Therefore, the first-order moment of $Q_{i}$ can be formulated as

$$
\mathrm{E}[Q]=\frac{1}{2}\left(\mathrm{E}\left[Y^{2}\right]+4 \mathrm{E}^{2}[Y]+\mathrm{E}[Y]\right)
$$

where $\mathrm{E}[Y]$ and $\mathrm{E}\left[Y^{2}\right]$ are obtained by Eqs. (20) and (21), respectively. As a result, based on Eqs. (23), (24) and (26), the average uplink AoI can be rewritten as

$$
\bar{\Delta}_{U}=\frac{1}{2}\left(\frac{\mathrm{E}\left[Y^{2}\right]}{\mathrm{E}[Y]}+4 \mathrm{E}[Y]+1\right) .
$$

Remark 1 : Observing from Eq. (27), for the case of $P_{r} \rightarrow \infty$ and that of $P_{r} \rightarrow 0$, the average AoI gradually approaches to $\bar{\Delta}_{U} \rightarrow \infty$.

\section{Average AoI Minimisation}

Note that the average AoI in the WPRCN depends on two factors related to the relay's transmit power/equivalent average power consumption $P_{r}$, namely the first-order moment $\mathrm{E}[X]$ for the recharging time at the relay and the joint successful 
transmission probability $\pi$. Given $r$, by increasing $P_{r}$, E $[X]$ increases resulting in a higher AoI, while $\pi$ increases, as well, resulting in a lower AoI. Hence, there is an optimal $P_{r}^{*}$ to minimise the average AoI. The optimisation problem is then formulated as

$$
\text { (P1): } P_{r}^{*}=\arg \min _{P_{r}>0} \bar{\Delta}_{U} .
$$

To get the solution of (P1), the successful transmission probability $\pi$ in Eq. (22) should be further formulated. However, the formulation of $\pi$ varies with DF and AF protocols. Moreover, it is not straightforward to derive a closed-form solution to (P1), which is then solved numerically. It is detailed in Sections IV and $\mathrm{V}$, respectively.

\section{Average AoI in the WPRCN with DF Protocol}

\section{A. Average AoI with An Ideal DF Protocol}

1) Successful Transmission Probability: Let $X_{1}$ and $X_{2}$ represent two independent random variables following an exponential distribution with a parameter $\lambda$. According to Eqs. (4), (7) and (22), the joint successful transmission probability in the uplink transmission can be written as follows

$$
\begin{aligned}
& \pi=\mathcal{P}\left\{\left(\log \left(1+S_{u 1 k_{1}}\right) \geq r\right) \cap\left(\log \left(1+S_{i d f, k_{2}}\right) \geq r\right)\right\} \\
& =\mathcal{P}\left\{\left(X_{1} \geq \frac{\left(2^{r}-1\right) \sigma_{0}^{2}}{P_{s} \Omega_{u 1}^{-1}}\right) \bigcap\left(X_{2} \geq \frac{\left(2^{r}-1\right) \sigma_{0}^{2}}{P_{r} \Omega_{u 2}^{-1}}\right)\right\} \\
& =\int_{\frac{\left(2^{r}-1\right) \Omega_{u 1} \sigma_{0}^{2}}{P_{s}}}^{\infty} \lambda e^{-\lambda x_{1}} d x_{1} \int_{\frac{\left(2^{r}-1\right) \Omega_{u \sigma^{2}} \sigma_{0}^{2}}{P_{r}}}^{\infty} \lambda e^{-\lambda x_{2}} d x_{2} \\
& =e^{-\lambda \sigma_{0}^{2}\left(2^{r}-1\right) \cdot\left(\frac{\Omega_{u 1}}{P_{s}}+\frac{\Omega_{u 2}}{P_{r}}\right)} .
\end{aligned}
$$

2) Average AoI Minimisation: The average uplink AoI is a differentiable function with respect to the relay's transmit power $P_{r}$ within the range of $P_{r}>0$. According to Remark $l$ and to the fact that the average AoI is finite when $P_{r}$ is any finite positive value, the gradient descent (GD) method detailed in [40] can be relied upon for solving (P1), where the search direction is the negative gradient $\partial P_{r}=-\frac{\mathrm{d} \bar{\Lambda}_{U}}{\mathrm{~d} P_{r}}$. The gradient of $\bar{\Delta}_{U}$ is calculated by the following steps:

- Step 1: Get the gradient of $\pi$ and $\theta$ as

$$
\begin{aligned}
\pi^{\prime} & =\frac{\mathrm{d} \pi}{\mathrm{d} P_{r}}=\frac{\lambda\left(2^{r}-1\right) \Omega_{u 2} \sigma_{0}^{2}}{P_{r}^{2}} \cdot e^{\left(-\frac{\lambda\left(2^{r}-1\right) \Omega_{u 2} \sigma_{0}^{2}}{P_{r}}\right)}, \\
\theta^{\prime} & =\frac{\mathrm{d} \theta}{\mathrm{d} P_{r}}=\frac{\lambda \Omega_{d}}{\zeta P_{t}} .
\end{aligned}
$$

- Step 2: Get the gradient of $\mathrm{E}[Y]$ and $\mathrm{E}\left[Y^{2}\right]$ as

$$
\begin{aligned}
& \mathrm{E}[Y]^{\prime}=\frac{\mathrm{d}[Y]}{\mathrm{d} P_{r}}=\frac{\theta^{\prime} \pi-(1+\theta) \pi^{\prime}}{\pi^{2}}, \\
& \mathrm{E}\left[Y^{2}\right]^{\prime}=\frac{\mathrm{dE}\left[Y^{2}\right]}{\mathrm{d} P_{r}}=\frac{\pi^{2} \gamma_{1}+\pi \gamma_{2}-\gamma_{3}}{\pi^{3}},
\end{aligned}
$$

where we have $\gamma_{1}=1+3 \theta^{\prime}-2 \theta^{\prime} \theta, \gamma_{2}=\left(\theta^{2}-3 \theta+1\right) \pi^{\prime}+4 \theta^{\prime} \theta$ and $\gamma_{3}=4 \pi^{\prime}\left(1+\theta^{2}\right)$.

- Step 3: Get the gradient of $\bar{\Delta}_{U}$ as

$$
\frac{\mathrm{d} \bar{\Delta}_{U}}{\mathrm{~d} P_{r}}=\frac{1}{2}\left(\frac{\mathrm{E}\left[Y^{2}\right]^{\prime}}{\mathrm{E}[Y]}-\frac{\mathrm{E}\left[Y^{2}\right] \mathrm{E}[Y]^{\prime}}{\mathrm{E}^{2}[Y]}+4 \mathrm{E}[Y]^{\prime}\right) .
$$

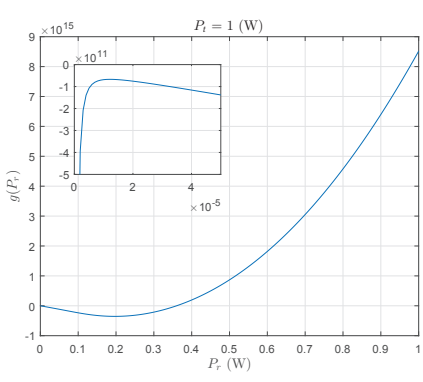

(a)

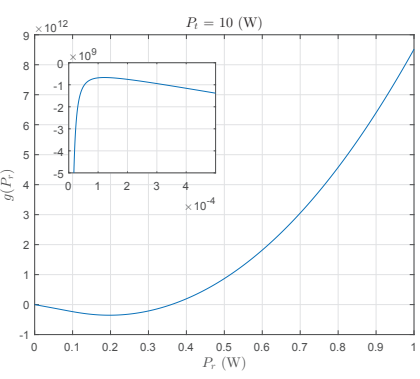

(b)
Fig. 3. Numerical calculation of the function $g\left(P_{r}\right)$ with $d_{1}=d_{2}=10 \mathrm{~m}$, when we have (a) $P_{t}=1 \mathrm{~W}$ and (b) $P_{t}=10 \mathrm{~W}$.

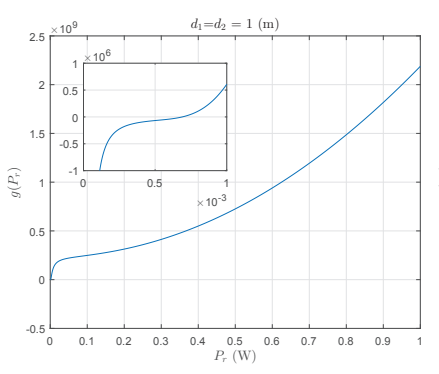

(a)

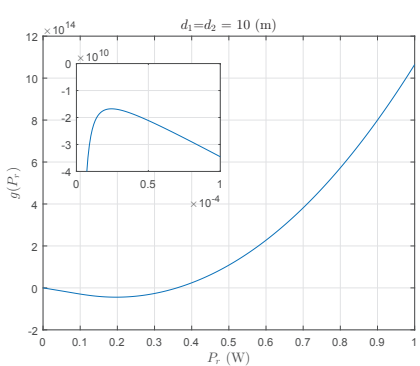

(b)
Fig. 4. Numerical calculation of the function $g\left(P_{r}\right)$ with $P_{t}=2 \mathrm{~W}$, when we have (a) $d_{1}=d_{2}=1 \mathrm{~m}$ and (b) $d_{1}=d_{2}=10 \mathrm{~m}$.

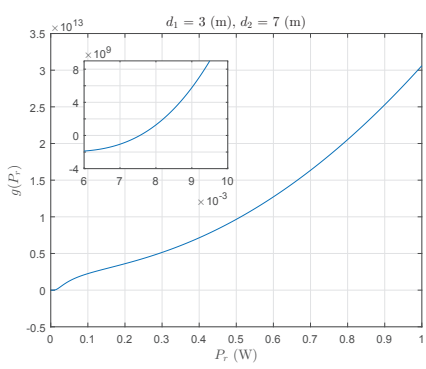

(a)

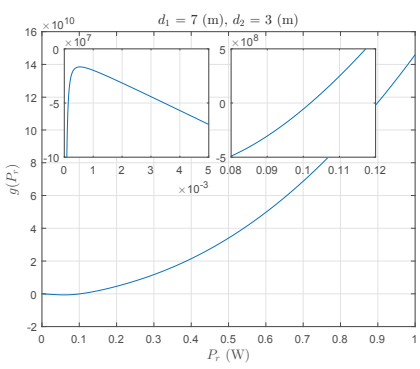

(b)
Fig. 5. Numerical calculation of the function $g\left(P_{r}\right)$ with a total transmission distance $d_{1}+d_{2}=10 \mathrm{~m}$, when we have (a) $d_{1}=3 \mathrm{~m}, d_{2}=7 \mathrm{~m}$ and (b) $d_{1}=7 \mathrm{~m}, d_{2}=3 \mathrm{~m}$.

By updating the transmit power of the relay as $P_{r}=$ $P_{r}+t \partial P_{r}$, where $t$ is the step size, we search for a $P_{r}^{*}$ satisfying $\left\|\frac{\mathrm{d} \bar{\Delta}_{U}}{\mathrm{~d} P_{r}}\right\| \leq \eta$, where $\eta$ is an arbitrarily small and positive threshold. The corresponding $\bar{\Delta}_{U}^{*}$ is the minimum average AoI.

Discussion on the average AoI $\bar{\Delta}_{U}$ : The average AoI $\bar{\Delta}_{U}$ firstly reduces to the minimum and it then increases, as we increase the relay's transmit power $P_{r}$. This is because a higher $P_{r}$ can facilitate the successful update transmission in the 2nd-hop. However, as we continuously increase $P_{r}$, the capacitor of the relay may be quickly drained. Therefore, it requires more transmission blocks to be fully recharged again. During the recharging, the relay cannot forward any update to the H-AP, which may substantially increase the average AoI. According to this physical truth, we know that the average AoI $\bar{\Delta}_{U}$ should be a convex function (or should have only one minimum extremum) with respect to $P_{r}$ in practice.

From the perspective of algorithm, the GD method can 
guarantee a global optimal solution when the optimisation problem is convex. Moreover, it can still find the global optimal solution, when the objective function $\bar{\Delta}_{U}$ simultaneously satisfies the following two conditions: (1) it has no stagnation point, (2) it only has a single minimum extremum. Specifically, the derivative of the average AoI $\bar{\Delta}_{U}$ with respect to $P_{r}$ can be formulated as Eq. (35) in page 8, where we define the following four constants $\eta_{1} \triangleq \frac{\lambda \Omega_{d}}{\zeta P_{t} T_{b}}, \eta_{2} \triangleq \frac{\lambda \sigma_{0}^{2}\left(2^{r}-1\right) \Omega_{u 1}}{P_{s}}$, $\eta_{3} \triangleq e^{-\eta_{2}}$ and $\eta_{4} \triangleq \lambda \sigma_{0}^{2}\left(2^{r}-1\right) \Omega_{u 2}$. By following the parameter setting in TABLE I of Section VI, we make the following approximations $\eta_{3} \approx 1$ and $\eta_{4} \approx 0$. The derivative $\frac{\mathrm{d} \bar{\Delta}_{U}}{\mathrm{~d} P_{r}}$ of the average AoI with respect to $P_{r}$ is then formulated as Eq. (36) in the next page. Since $\frac{\eta_{4}}{P_{r}}$ exits in Eq. (36), $\frac{\mathrm{d} \bar{\Delta}_{U}}{\mathrm{~d} P_{r}}$ is analysed by comparing $P_{r}$ to $\eta_{4}$ in the following two cases, namely $P_{r} \geq 1$ $\mathrm{W}$ and $P_{r}<1 \mathrm{~W}$.

In the case of $P_{r} \geq 1 \mathrm{~W}$, we have $\frac{\eta_{4}}{P_{r}} \approx 0$. Therefore, Eq. (36) can be reformulated as

$$
\frac{\mathrm{d} \bar{\Delta}_{U}}{\mathrm{~d} P_{r}}=\frac{10 \eta_{1}^{2} P_{r}+8 \eta_{1}^{3} P_{r}^{2}+\eta_{1} P_{r}+\gamma_{1}^{3}+10 \eta_{1}+1}{2\left(1+\eta_{1} P_{r}^{2}\right)} .
$$

Obviously, we have $\frac{\mathrm{d} \bar{\Delta}_{U}}{\mathrm{~d} P_{r}}>0$, which indicates that the average AoI $\bar{\Delta}_{U}$ is a monotonously increasing function with respect to $P_{r}$ when we have $P_{r} \geq 1 \mathrm{~W}$. Therefore, the extreme point of $\bar{\Delta}_{U}$ with $\frac{\mathrm{d} \bar{\Delta}_{U}}{\mathrm{~d} P_{r}}=0$ must exist, when we have $P_{r} \in(0,1) \mathrm{W}$.

In the case of $P_{r}<1 \mathrm{~W}$, the ratio $\frac{\eta_{4}}{P_{r}}$ cannot be approximated to zero, when $P_{r}$ is close to $\eta_{4}$. Unfortunately, $\frac{\mathrm{d} \bar{\Delta}_{U}}{\mathrm{~d} P_{r}}$ in Eq. (36) is a sophisticated transcendental function. We cannot theoretically find solutions to let $\frac{\mathrm{d} \bar{\Delta}_{U}}{\mathrm{~d} P_{r}}=0$. Therefore, we have to exploit numerical calculation here. By letting $\frac{\mathrm{d} \bar{\Delta}_{U}}{\mathrm{~d} P_{r}}=0$, Eq. (36) can be simplified to $g\left(P_{r}\right)$, which is defined in Eq. (38) in the next page. Given the parameters in TABLE I, we can only find a single solution $P_{r}^{*}$ to let $g\left(P_{r}\right)=0$, as portrayed in Figs. 3, 4 and 5. Moreover, we have $g\left(P_{r}\right)<0$ when $P_{r} \in\left(0, P_{r}^{*}\right)$ and $g\left(P_{r}\right)>0$ when $P_{r} \in\left(P_{r}^{*}, \infty\right)$. As a result, GD method can obtain the global optimal solution to (P1), since $\bar{\Delta}_{U}$ has no stagnation point and it only has a single minimum, when the parameter setting follows TABLE I.

\section{B. The WPRCN with A Practical DF Protocol}

1) Successful Transmission Probability: The following Theorem 1 is proposed to formulate the successful transmission probability $\pi$.

Theorem 1: By exploiting the Taylor approximation, the successful transmission probability with a practical DF protocol is expressed as Eq. (39) in the next page, where $S_{p d f, k}$ is given by Eq. (11) and we have $m_{l}=\left(\gamma_{l+1}+\gamma_{l}\right) / 2, \alpha_{1}=P_{s} \Omega_{u 1}^{-1}$, $\alpha_{2}=\sigma_{0}^{4} \Omega_{u 2}\left(2^{r}-1\right) / a$ as well as $\alpha_{3}=P_{r} \sigma_{0}^{2} / a$. We define $f\left(x_{1}\right)$ as the original integrand, as shown in Eq. (48). When $N=4$, the different-order derivatives of $f\left(x_{1}\right)$ with respect to $x_{1}$ at the point $m_{l}$ is expressed as Eqs. (40)-(43) in the next page.

Proof: Please refer to Appendix A for the detailed proof.

Discussion on Theorem 1: We utilise the Taylor expansion to approximate the original integrand of the successful transmission probability, as shown in Eq. (48). However, the accuracy

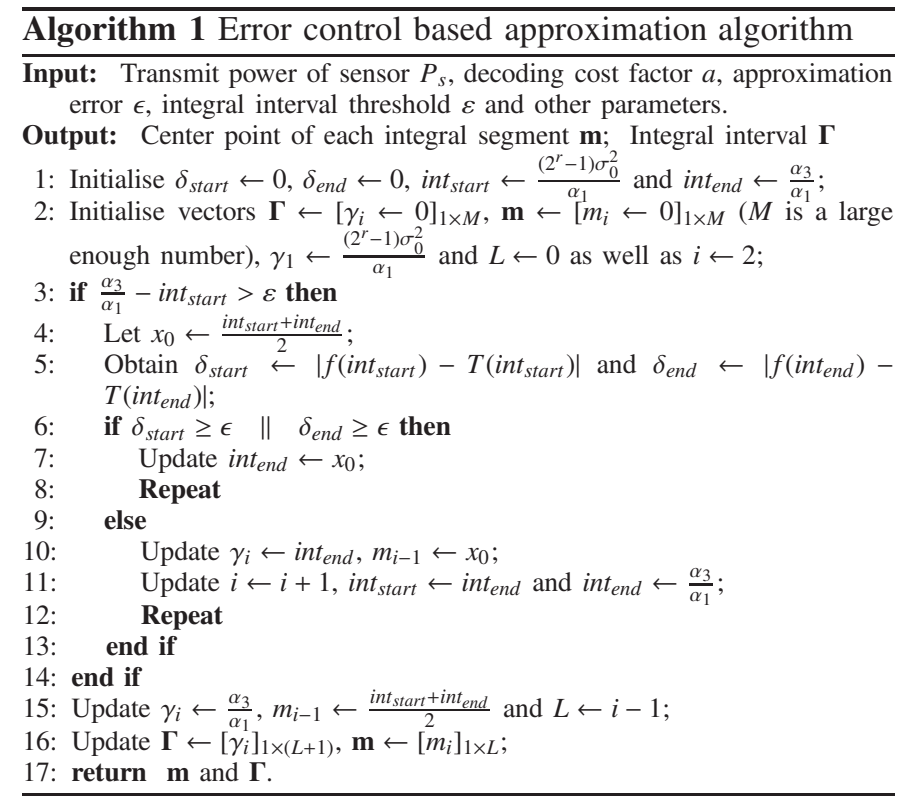

of the approximation may be reduced if the integral interval is increased. Therefore, we split the integral interval into $L$ sections for improving the approximation accuracy. Note that the $l$-th section is centered at the bisection point $m_{l}$ of the integral interval $\left[\gamma_{l}, \gamma_{l+1}\right]$.

Furthermore, an error control based algorithm is proposed in order to determine the integral intervals $\boldsymbol{\Gamma}=\left[\gamma_{i}\right]_{1 \times(L+1)}$ and the corresponding bisection points $\mathbf{m}=\left[m_{i}\right]_{1 \times L}$, which is detailed in the pseudo code of Algorithm 1. The main steps of this algorithm is summarised as below:

- Step 1: Define int start $_{\text {and }}$ ant $t_{\text {end }}$ as the start and the end points of the integral interval, respectively. Initialise the differences between the original function $f\left(x_{1}\right)$ in Eq. (48) and the approximation $T\left(x_{1}\right), \delta_{\text {start }}$ and $\delta_{\text {end }}$. Define vectors $\boldsymbol{\Gamma}$ and $\mathbf{m}$ for recording the searched integral intervals and the corresponding bisection points separately, as shown in Lines 1 and 2 of Algorithm 1.

- Step 2: Initialise the bisection point $x_{0}$ of the integral interval. Obtain $\delta_{\text {start }}$ and $\delta_{\text {end }}$. If either of them does not satisfy the approximation error $\epsilon$, update $\delta_{\text {end }}$ to $x_{0}$ and go to Step 3. Otherwise, the corresponding entries $\gamma_{i}$ and $m_{i-1}$ are updated to int $t_{\text {end }}$ and $x_{0}$, respectively. Then, update the integral interval $\left[\right.$ int $_{\text {start }}$, int $\left._{\text {end }}\right]$. This step is characterised in Lines 4-13 of Algorithm 1. We approximate the remaining intervals by going to Step 3 .

- Step 3: If the difference of the integral upper bound between the original function $f\left(x_{1}\right)$ and the approximation $T\left(x_{1}\right)$ is higher than a pre-defined threshold $\varepsilon$, repeat Step 2 , as shown in Line 3 of Algorithm 1. Otherwise, update $\gamma_{i}$ and $m_{i-1}$ as well as the number of the integral intervals $L$ and update the dimensions of $\boldsymbol{\Gamma}$ and $\mathbf{m}$ to $(L+1)$ and $L$, respectively, as shown in Lines 15 and 16 of Algorithm 1.

By discretizing the integral interval into $n$ points, we have $n / 2$ searches and the complexity of $i$-th search is $O\left(\log _{2}(n-2 i+2)\right)$. Therefore, the complexity of Algorithm 1 is $O\left(\sum_{i=1}^{n / 2} \log _{2}(n-2 i+2)\right)$. 


$$
\begin{aligned}
\frac{\mathrm{d} \bar{\Delta}_{U}}{\mathrm{~d} P_{r}}= & {\left[\left(\left(1+3 \eta_{1}\right) \eta_{3}^{2} \eta_{1}-5 \eta_{1}^{2} \eta_{3}^{2}\right) P_{r}-2 \eta_{1}^{3} \eta_{3}^{2} P_{r}^{2}+\eta_{3}^{2}+4 \eta_{1} \eta_{3}^{2}+\eta_{1}^{3} \eta_{3}^{2}\right] \frac{1}{2 \eta_{3}^{2}\left(1+\eta_{1} P_{r}\right)^{2}} } \\
& +\left[\left(12 \eta_{1}^{2} \eta_{3}-6 \eta_{1}^{3} \eta_{4}\right) P_{r}+10 \eta_{1}^{3} \eta_{3} P_{r}^{2}-\frac{6 \eta_{4}}{P_{r}^{2}}-\frac{14 \eta_{1} \eta_{4}}{P_{r}}+6 \eta_{1} \eta_{3}-14 \eta_{1}^{2} \eta_{4}\right] \frac{1}{2 \eta_{3}^{2}\left(1+\eta_{1} P_{r}\right)^{2} e^{-\frac{\eta_{4}}{P_{r}}}} \\
& -\left(\frac{4 \eta_{4}}{P_{r}^{2}}+\frac{4 \eta_{1} \eta_{4}}{P_{r}}+4 \eta_{1}^{3} \eta_{4} P_{r}+4 \eta_{1}^{2} \eta_{4}\right) \frac{1}{2 \eta_{3}^{2}\left(1+\eta_{1} P_{r}\right)^{2} e^{-2 \frac{\eta_{4}}{P_{r}}}},
\end{aligned}
$$

$$
\begin{aligned}
\frac{\mathrm{d} \bar{\Delta}_{U}}{\mathrm{~d} P_{r}}= & {\left[\left(1-2 \eta_{1}\right) \eta_{1} P_{r}-2 \eta_{1}^{3} P_{r}^{2}+\eta_{1}^{3}+4 \eta_{1}+1\right] \frac{1}{2\left(1+\eta_{1} P_{r}\right)^{2}}-\left(\frac{4 \eta_{4}}{P_{r}^{2}}+\frac{4 \eta_{1} \eta_{4}}{P_{r}}\right) \frac{1}{2 e^{-2 \frac{\eta_{4}}{P_{r}}}\left(1+\eta_{1} P_{r}\right)^{2}} } \\
& +\left[12 \eta_{1}^{2} P_{r}+10 \eta_{1}^{3} P_{r}^{2}-\frac{6 \eta_{4}}{P_{r}^{2}}-\frac{14 \eta_{1} \eta_{4}}{P_{r}}+6 \eta_{1}\right] \frac{1}{2 e^{-\frac{\eta_{4}}{P_{r}}}\left(1+\eta_{1} P_{r}\right)^{2}} .
\end{aligned}
$$

$$
\begin{aligned}
g\left(P_{r}\right) \triangleq & {\left[\left(1-2 \eta_{1}\right) \eta_{1} P_{r}-2 \eta_{1}^{3} P_{r}^{2}+\eta_{1}^{3}+4 \eta_{1}+1\right] e^{-2 \frac{\eta_{4}}{P_{r}}} } \\
& +\left[12 \eta_{1}^{2} P_{r}+10 \eta_{1}^{3} P_{r}^{2}-\frac{6 \eta_{4}}{P_{r}^{2}}-\frac{14 \eta_{1} \eta_{4}}{P_{r}}+6 \eta_{1}\right] e^{-\frac{\eta_{4}}{P_{r}}}-\left(\frac{4 \eta_{4}}{P_{r}^{2}}+\frac{4 \eta_{1} \eta_{4}}{P_{r}}\right)=0 .
\end{aligned}
$$

$$
\pi=\mathcal{P}\left\{\log \left(1+S_{u 1 k_{1}}\right) \geq r \cap \log \left(1+S_{p d f, k_{2}}\right) \geq r\right\} \approx \sum_{l=1}^{L}\left[f\left(m_{l}\right)\left(\gamma_{l+1}-\gamma_{l}\right)+\sum_{n=1}^{N} \frac{f^{(n)}\left(m_{l}\right)}{(n+1) !}\left(\left(\gamma_{l+1}-m_{l}\right)^{n+1}-\left(\gamma_{l}-m_{l}\right)^{n+1}\right)\right]
$$

$$
\begin{aligned}
f^{(1)}\left(m_{l}\right) & =-\lambda^{2} e^{-\lambda\left(m_{l}+\frac{\alpha_{2}}{\alpha_{3}-\alpha_{1} m_{l}}\right)}\left(1+\frac{\alpha_{1} \alpha_{2}}{\left(\alpha_{3}-\alpha_{1} m_{l}\right)^{2}}\right) \\
f^{(2)}\left(m_{l}\right) & =\lambda e^{-\lambda\left(m_{l}+\frac{\alpha_{2}}{\alpha_{3}-\alpha_{1} m_{l}}\right)}\left[\lambda^{2}\left(1+\frac{\alpha_{1} \alpha_{2}}{\left(\alpha_{3}-\alpha_{1} m_{l}\right)^{2}}\right)^{2}-\frac{2 \alpha_{1}^{2} \alpha_{2} \lambda}{\left(\alpha_{3}-\alpha_{1} m_{l}\right)^{3}}\right] \\
f^{(3)}\left(m_{l}\right) & =e^{-\lambda\left(m_{l}+\frac{\alpha_{2}}{\alpha_{3}-\alpha_{1} m_{l}}\right)}\left[-\lambda^{4}\left(1+\frac{\alpha_{1} \alpha_{2}}{\left(\alpha_{3}-\alpha_{1} m_{l}\right)^{2}}\right)^{3}+\frac{6 \alpha_{1}^{2} \alpha_{2} \lambda^{3}}{\left(\alpha_{3}-\alpha_{1} m_{l}\right)^{3}}\left(1+\frac{\alpha_{1} \alpha_{2}}{\left(\alpha_{3}-\alpha_{1} m_{l}\right)^{2}}\right)-\frac{6 \alpha_{1}^{3} \alpha_{2} \lambda^{2}}{\left(\alpha_{3}-\alpha_{1} m_{l}\right)^{4}}\right] \\
f^{(4)}\left(m_{l}\right) & =\lambda e^{-\lambda\left(m_{l}+\frac{\alpha_{2}}{\alpha_{3}-\alpha_{1} m_{l}}\right)}\left[\lambda^{4}\left(1+\frac{\alpha_{1} \alpha_{2}}{\left(\alpha_{3}-\alpha_{1} m_{l}\right)^{2}}\right)^{4}-\frac{12 \alpha_{1}^{2} \alpha_{2} \lambda^{3}}{\left(\alpha_{3}-\alpha_{1} m_{l}\right)^{3}}\left(1+\frac{\alpha_{1} \alpha_{2}}{\left(\alpha_{3}-\alpha_{1} m_{l}\right)^{2}}\right)^{2}+\frac{24 \alpha_{1}^{3} \alpha_{2} \lambda^{2}}{\left(\alpha_{3}-\alpha_{1} m_{l}\right)^{4}}\right] \\
& -\lambda e^{-\lambda\left(m_{l}+\frac{\alpha_{2}}{\alpha_{3}-\alpha_{1} m_{l}}\right)}\left[\frac{24 \alpha_{1}^{4} \alpha_{2} \lambda}{\left(\alpha_{3}-\alpha_{1} m_{l}\right)^{5}}-\frac{36 \alpha_{1}^{4} \alpha_{2}^{2} \lambda^{2}}{\left(\alpha_{3}-\alpha_{1} m_{l}\right)^{6}}\right]
\end{aligned}
$$

2) Average AoI Minimisation: Since $\pi$ is a function of $P_{r}$, the problem (P1) can be solved by a one dimensional search, such as a golden-section method [41].

\section{Average AoI in the WPRCN with AF Protocol}

According to $S_{a f, k}$ in Eq. (14), the spectral efficiency constraint in the 2nd-hop can be derived as

$$
\begin{aligned}
& \log \left(1+S_{a f, k}\right) \geq r \\
\Rightarrow & P_{s} P_{r} \Omega_{u 1}^{-1} \Omega_{u 2}^{-1}\left|h_{u 1}\right|^{2}\left|h_{u 2}\right|^{2} \geq \\
& \left(2^{r}-1\right) \sigma_{0}^{2}\left(P_{s}\left|h_{u 1}\right|^{2} \Omega_{u 1}^{-1}+P_{r}\left|h_{u 2}\right|^{2} \Omega_{u 2}^{-1}\right) \\
\Rightarrow & \frac{P_{s} \Omega_{u 1}^{-1}}{\left|h_{u 2}\right|^{2}}+\frac{P_{r} \Omega_{u 2}^{-1}}{\left|h_{u 1}\right|^{2}} \leq \frac{P_{s} P_{r} \Omega_{u 1}^{-1} \Omega_{u 2}^{-1}}{\left(2^{r}-1\right) \sigma_{0}^{2}} .
\end{aligned}
$$

Let $X_{1}$ and $X_{2}$ denote exponentially distributed independent random variables with the same parameter $\lambda$. The successful transmission probability $\pi$ in the 2 nd-hop can be calculated as

$$
\begin{aligned}
\pi & =\mathcal{P}\left\{\log \left(1+S_{a f, k}\right) \geq r\right\}, \\
& =\mathcal{P}\left\{\left(X_{1} \geq \frac{\mu_{1}}{\mu_{3}}\right) \bigcap\left(X_{2} \geq \frac{\mu_{2}}{\mu_{3}-\frac{\mu_{1}}{X_{1}}}\right)\right\}, \\
& =\int_{\frac{\mu_{1}}{\mu_{3}}}^{\infty} \lambda e^{-\lambda x_{1}} \int_{\frac{\mu_{2} x_{1}}{\mu_{3} x_{1}-\mu_{1}}}^{\infty} \lambda e^{-\lambda x_{2}} d x_{2} d x_{1}, \\
& =\lim _{t \rightarrow \frac{\mu_{1}}{\mu_{3}}} \int_{t}^{\infty} \lambda e^{-\lambda\left(x_{1}+\frac{\mu_{2} x_{1}}{\mu_{3} x_{1}-\mu_{1}}\right)} d x_{1}, \\
& =\lim _{t_{1} \rightarrow 0} \lim _{t_{2} \rightarrow \frac{\mu_{3}}{\mu_{1}}} \int_{t_{1}}^{t_{2}} \frac{\lambda}{y^{2}} e^{-\lambda\left(\frac{1}{y}+\frac{\mu_{2}}{\mu_{3}-\mu_{1} y}\right)} d y,
\end{aligned}
$$


TABLE I

Parameter SetTing

\begin{tabular}{lll}
\hline Parameter & Denotation & Value \\
\hline Transmit power of H-AP & $P_{t}$ & $4 \mathrm{~W}$ \\
Transmit power of sensor & $P_{s}$ & $5 * 10^{-4} \mathrm{~W}$ \\
Distance from the sensor to the relay & $d_{1}$ & $5 \mathrm{~m}$ \\
Noise power & $\sigma_{0}^{2}$ & $-50 \mathrm{dBm}$ \\
Distance from the relay to the H-AP & $d_{2}$ & $5 \mathrm{~m}$ \\
Spectral efficiency threshold & $r$ & $0.2 \mathrm{BPCU}$ \\
Path-loss reference distance & $d_{0}$ & $1 \mathrm{~m}$ \\
Path-loss exponent & $\alpha$ & 2 \\
Path-loss at reference distance & $\Omega_{0}$ & $30 \mathrm{~dB}$ \\
Decoding cost factor & $a$ & $10^{-8}$ \\
Rate parameter & $\lambda$ & 1 \\
Energy conversion efficiency & $\zeta$ & 0.8 \\
\hline
\end{tabular}

where we have $y=\frac{1}{x_{1}}, \mu_{1}=P_{r} \Omega_{u 2}^{-1}, \mu_{2}=P_{s} \Omega_{u 1}^{-1}$ and $\mu_{3}=$ $\frac{P_{s} P_{r} \Omega_{u 1}^{-1} \Omega_{u 2}^{-1}}{\left(2^{r}-1\right) \sigma_{0}^{2}}$.

A similar method to Algorithm 1 can be relied upon for obtaining $\pi$ with a fixed $P_{r}$. Then, the problem (P1) can be solved by a one-dimensional search.

\section{Numerical Results}

Apart from the uncorrelated block Rayleigh-fading [36], the path-loss $\Omega_{u 1}$ in the 1st-hop channel and $\Omega_{u 2}$ in the 2nd-hop channel can be formulated as $\Omega_{u i}=\Omega_{0} \cdot\left(\frac{d_{i}}{d_{0}}\right)^{\alpha}$ for $\forall i=1,2$. Similarly, the path-loss $\Omega_{d}$ during downlink WPT is formulated as $\Omega_{d}=\Omega_{0} \cdot\left(\frac{d_{2}}{d_{0}}\right)^{\alpha}$. We set the bandwidth and the length of a transmission block to be normalised as $W=1$ $\mathrm{Hz}$ and $T_{b}=1 \mathrm{~s}$. Simulation parameters in both physical and MAC layers are in line with TABLE I, unless some parameter changes are particularly mentioned.

\section{A. Performance of Taylor Approximation}

In order to verify the performance of Algorithm 1 based on Taylor approximation, we plot the original functions and their approximation in Fig. 6, where the original functions are $f(x)=\lambda e^{-\lambda\left(x+\frac{\alpha_{2}}{\alpha_{3}-\alpha_{1} x}\right)}$ and $f(y)=\frac{\lambda}{v^{2}} e^{-\lambda\left(\frac{1}{y}+\frac{\mu_{2}}{\mu_{3}-\mu_{1} y}\right)}$, respectively, according to Eqs. (47) and (45). Observe from both Fig. 6 (a) and (b) that the two curves almost coincide with each other, which confirms the approximation accuracy of Algorithm 1.

\section{B. Average AoI Analysis}

We plot the transmit power/equivalent average power consumption $P_{r}$ of the relay against the average AoI per transmission block in Fig. 7. By setting $T_{b}$ to $1 \mathrm{~s}$, we have $P_{r}=B$. Observe from Fig. 7 (a) and (b) that as $B$ increases, the average AoI firstly reduces to the minimum and it then increases. This is because, a higher $B$ facilitates the 2ndhop transmission owing to more available energy. However, as we continuously increase $B$, it requires more transmission blocks to recharge the relay, which dominantly degrades the average AoI performance. Therefore, an appropriate capacitor, which can store sufficient energy for update transmission and does not need a long time to be fully recharged, should be selected before being implemented to the batteryless relay by considering a specific wireless environment. Moreover,
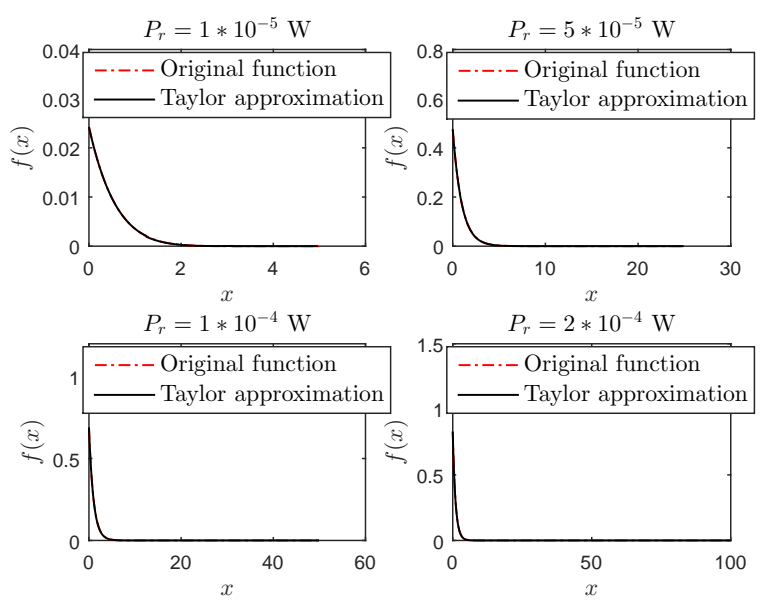

(a)
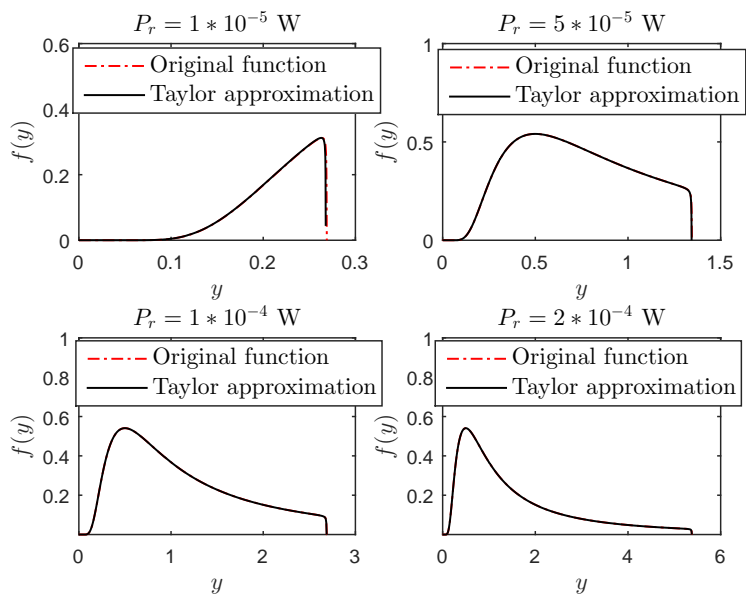

(b)

Fig. 6. Taylor approximation in the WPRCN (a) with a practical DF protocol and (b) with an AF protocol.

observe from Fig. 7 (a) and (b) that the relay close to the H-AP is also capable of reducing the average AoI. This is because the resultant path-loss can be reduced and the WPT performance from the H-AP to the relay can be substantially improved. Moreover, the optimal value of $P_{r}$ matches with the optimal solution obtained by solving (P1). Finally, we further verify the approximation performance of Algorithm 1 by comparing it to the Monte Carlo simulation. Observe from Fig. 7 (a) and (b) that these two methods achieve similar average AoI performance.

\section{Transmit Power of $H-A P$}

Fig. 8 depicts the average AoI minimisation versus the $\mathrm{H}$ AP's transmit power $P_{t}$, where the factor of the decoding cost is set to $a=5 \times 10^{-5}$ [39] and the spectral efficiency threshold is set to $r=0.5$ BPCU. Observe from Fig. 8 that the average AoI of the WPRCN with different relaying protocols are substantially decreased as we increase $P_{t}$. This is because a higher transmit power of the H-AP substantially improves the downlink WPT performance towards the relay. Therefore, the capacitor of the relay can be rapidly recharged. 


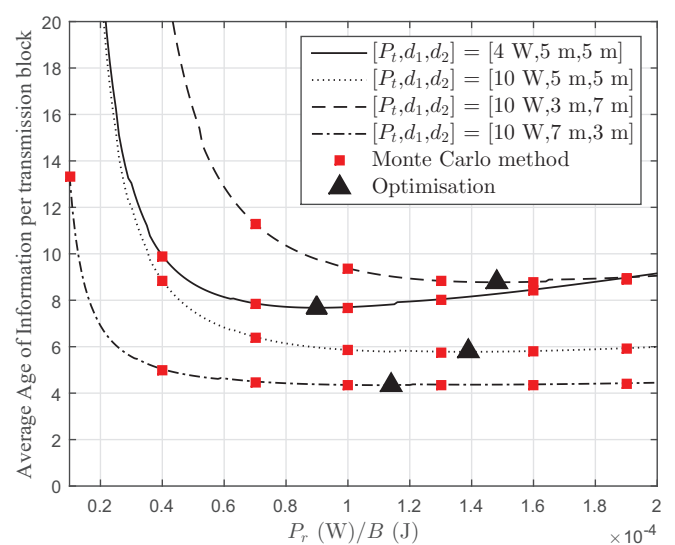

(a)

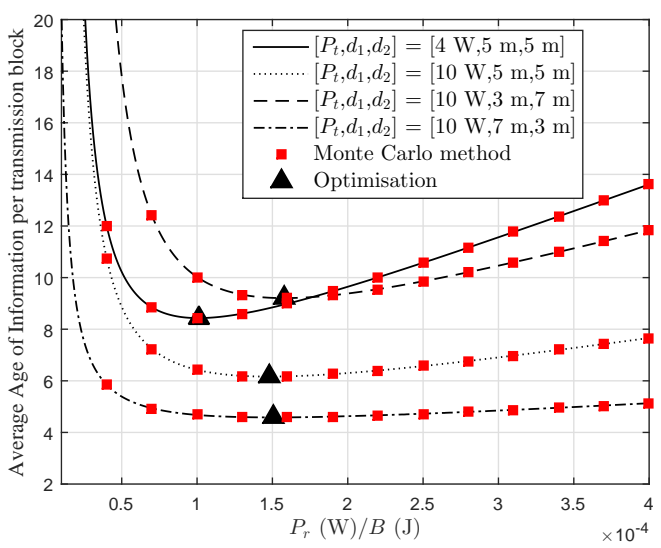

(b)

Fig. 7. Average AoI analysis of the WPRCNs with (a) a practical DF protocol and (b) an AF protocol.

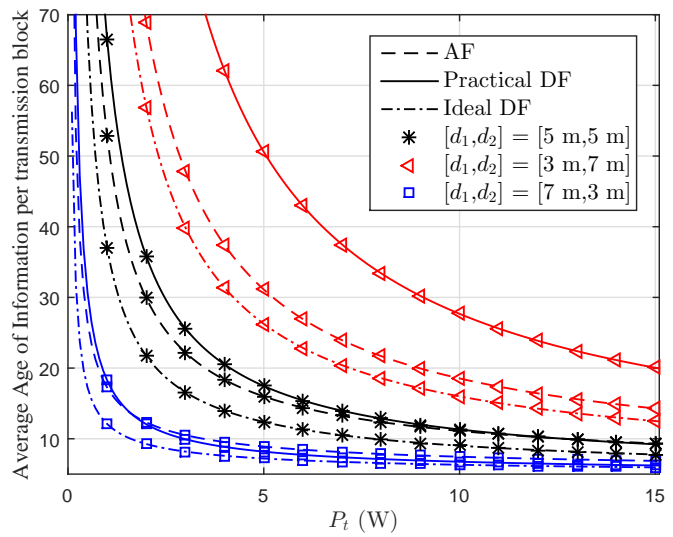

Fig. 8. Impact of the transmit power of the H-AP on average AoI per transmission block.

Furthermore, the WPRCNs with an ideal DF and an AF protocols outperform that with a practical DF protocol, when the relay is far from the H-AP. As the relay gets closer to the H-AP, the performance gap between the practical DF protocol and the AF counterpart is reduced, while the DF protocol may achieve an even better performance than the AF with $P_{t}>1.7 \mathrm{~W}$ when we have $\left[d_{1}, d_{2}\right]=[7 \mathrm{~m}, 3 \mathrm{~m}]$. Furthermore, the performance gap between the WPRCN with

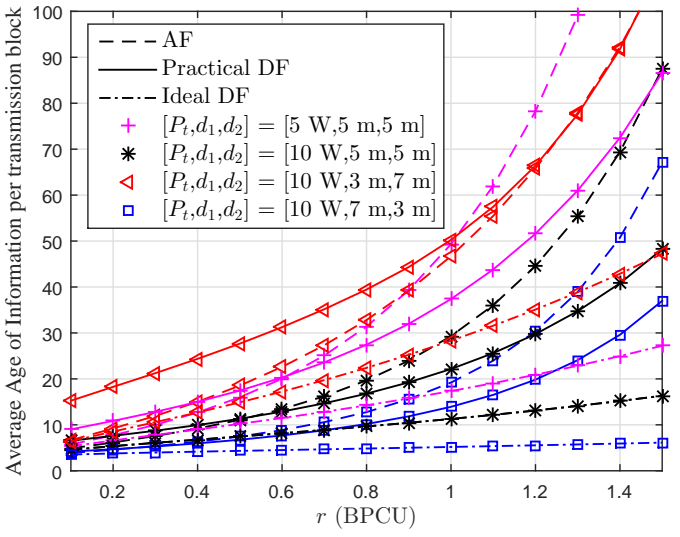

Fig. 9. Average AoI minimisation versus spectral efficiency threshold.

an ideal DF protocol and that with a practical DF protocol reduces. This is because the path-loss can be reduced and the WPT performance from the H-AP to the relay can be substantially improved so as to shorten the fully charging duration. Moreover, as $P_{t}$ increases, these two WPRCNs have similar performance, since more energy supply reduces the adverse impact of the decoding cost.

\section{Spectral Efficiency Constraint}

Fig. 9 plots the minimum average AoI versus the outage threshold $r$. The decoding cost $a$ is set to $5 \times 10^{-5}$. Observe from Fig. 9 that when $r$ increases, the average AoI of all the WPRCNs substantially increases. Furthermore, as $r$ increases, the performance gap between the practical DF protocol and the AF is enlarged. However, when the relay and the H-AP get further apart and $r$ reduces, the AoI performance of the WPRCNs with an AF protocol may become better than that with a practical DF protocol. This is because a shorter distance in the 1st-hop reduces the path-loss, which can considerably reduce the adverse impact of the 1st-hop noise amplified by the AF protocol aided relay.

\section{E. Transmission Distance in The 1st-And 2nd-Hops}

Fig. 10 plots the minimum average AoI versus distances $d_{1}$ and $d_{2}$ in the 1 st- and 2nd-hops. An increasing $d_{2}$ degrades the attainable average AoI performance of the both relaying protocols. This is because the increasing path-loss reduces the WPT performance. Observe from Fig. 10 (a), for a given $d_{2}$, the average AoI with a practical DF protocol considerably increases when we increase $d_{1}$. This is because an increasing $d_{1}$ worsens the uplink transmission in the 1st-hop, which reduces the successful transmission probability. Similarly, the average AoI with an AF protocol increases with an increasing $d_{1}$, as shown in Fig. 10 (b). This is because the path-loss in the 1st-hop is increased and the corresponding amplified noise is enlarged. Therefore, the successful transmission probability is degraded.

\section{Conclusion}

We studied the timeliness of a WPRCN with an H-AP, a wireless powered relay and a sensor by considering the longterm average AoI. Two different relaying protocols, i.e., AF 


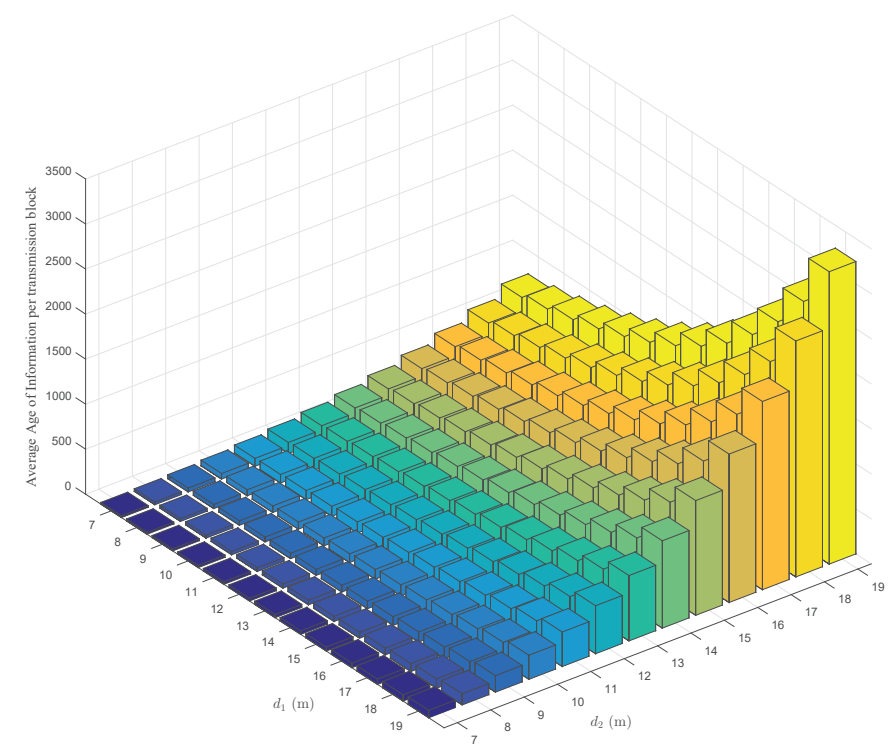

(a)

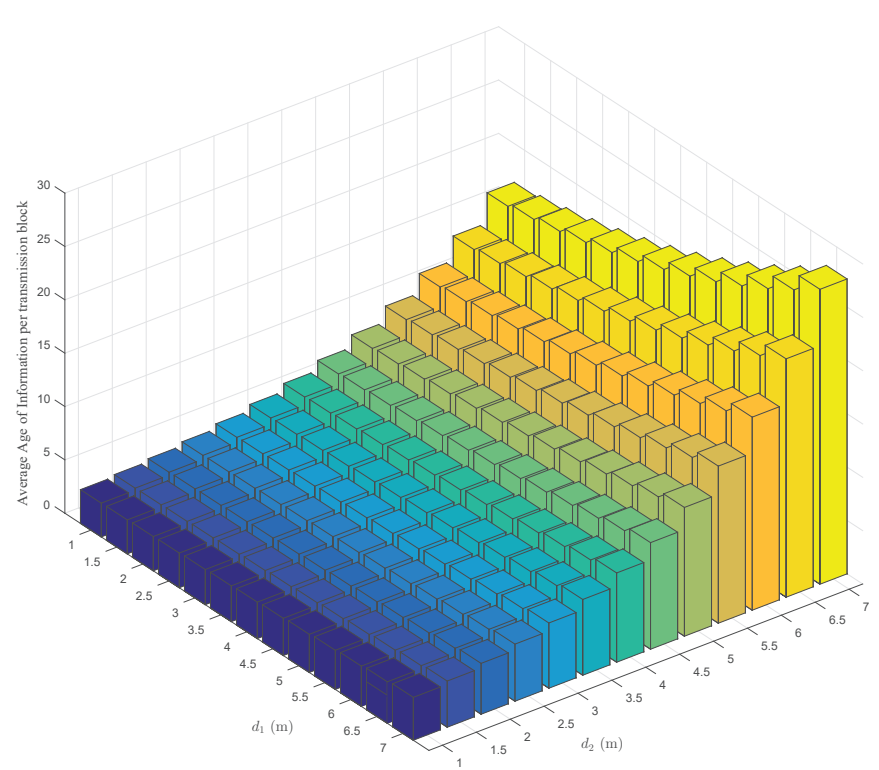

(b)

Fig. 10. Impact of 1st-hop and 2nd-hop transmission distance of the WPRCNs with (a) a practical DF protocol and (b) an AF protocol on average AoI per transmission block.

and DF, are considered in this paper, subject to the energy causality, while the impact of the decoding cost on the AoI performance with a practical DF protocol is investigated. A Taylor approximation based algorithm is proposed for enhancing approximation accuracy to a non-elementary function. The transmit power/the equivalent average power consumption of the relay is analysed and optimised to minimise the average AoI per transmission block in the WPRCN with different forwarding protocols. Our simulation results demonstrate the accuracy of the theoretical analysis, while the proposed algorithm is verified to achieve great approximation effect. Furthermore, we also find that in the case of sufficient energy supply, the AoI performance of the relay with a practical DF protocol outperforms that with an AF protocol, while the latter may have a better performance in the case of a low outagethreshold.

\section{APPENDIX A \\ Proof of Theorem 1}

A successful update transmission event in the 2nd-hop can be expressed as

$$
\begin{aligned}
& \log \left(1+S_{p d f, k}\right) \geq r \\
& \Rightarrow \frac{\left|h_{u 2 k}\right|^{2}\left(P_{r}-P_{d c, k}\right)}{\sigma_{0}^{2} \Omega_{u 2}} \geq 2^{r}-1 \\
& \Rightarrow P_{s} \Omega_{u 1}^{-1}\left|h_{u 1 k}\right|^{2}+\frac{\sigma_{0}^{4} \Omega_{u 2}\left(2^{r}-1\right)}{a\left|h_{u 2 k}\right|^{2}} \leq \frac{P_{r} \sigma_{0}^{2}}{a} .
\end{aligned}
$$

Let $X_{1}$ and $X_{2}$ represent two independent random variables following an exponential distribution with a parameter. The successful transmission probability in the 1st- and 2nd-hop can be formulated as

$$
\begin{aligned}
& \mathcal{P}\left\{\log \left(1+S_{u 1 k_{1}}\right) \geq r \cap \log \left(1+S_{p d f, k_{2}}\right) \geq r\right\} \\
& =\mathcal{P}\left\{\left(\frac{\left(2^{r}-1\right) \sigma_{0}^{2}}{\alpha_{1}} \leq X_{1} \leq \frac{\alpha_{3}}{\alpha_{1}}\right) \bigcap\left(X_{2} \geq \frac{\alpha_{2}}{\alpha_{3}-\alpha_{1} X_{1}}\right)\right\} \\
& =\int_{\frac{\left(2^{r}-1\right) \sigma_{0}^{2}}{\alpha_{1}}}^{\frac{\alpha_{3}}{\alpha_{1}}} \lambda e^{-\lambda x_{1}} \int_{\frac{\alpha_{2}}{\alpha_{3}-\alpha_{1} x_{1}}}^{\infty} \lambda e^{-\lambda x_{2}} \mathrm{~d} x_{2} \mathrm{~d} x_{1} \\
& =\lim _{t \rightarrow \frac{\alpha_{3}}{\alpha_{1}}-} \int_{\frac{\left(2^{r}-1\right) \sigma_{0}^{2}}{\alpha_{1}}}^{t} \lambda e^{-\lambda\left(x_{1}+\frac{\alpha_{2}}{\alpha_{3}-\alpha_{1} x_{1}}\right)} \mathrm{d} x_{1} .
\end{aligned}
$$

Since the integration in Eq. (47) cannot be transformed to an elementary function, Taylor expansion is exploited for reasonable approximation. Therefore, the original integrand $f\left(x_{1}\right)$ can be reformulated as

$$
\begin{aligned}
f\left(x_{1}\right) & =\lambda e^{-\lambda\left(x_{1}+\frac{\alpha_{2}}{\alpha_{3}-\alpha_{1} x_{1}}\right)} \\
& \approx f\left(x_{0}\right)+\sum_{n=1}^{N} \frac{f^{(n)}\left(x_{0}\right)}{n !}\left(x_{1}-x_{0}\right)^{n} \triangleq T\left(x_{1}\right),
\end{aligned}
$$

where the Taylor expansion with a limited number of terms is denoted as $T\left(x_{1}\right)$ centering at the point $x_{0}$. However, the approximation accuracy is reduced when the integral interval increases. We then divide the original function $f\left(x_{1}\right)$ into $L$ segments with different lengths, and integrate them segmentby-segment after the Taylor approximation. Hence, Eq. (47) can be reformulated as Eq. (49). When $N=4$, different-order derivative functions of $f\left(x_{1}\right)$ can be expressed as Eqs. (40)(43). Theorem 1 is proved.

\section{REFERENCES}

[1] X. Lu, P. Wang, D. Niyato, D. I. Kim, and Z. Han, "Wireless charging technologies: Fundamentals, standards, and network applications," IEEE Communications Surveys Tutorials, vol. 18, no. 2, pp. 1413-1452, Secondquarter 2016.

[2] J. Hu, K. Yang, G. Wen, and L. Hanzo, "Integrated data and energy communication network: A comprehensive survey," IEEE Communications Surveys Tutorials, vol. 20, no. 4, pp. 3169-3219, Fourthquarter 2018.

[3] M. Lei, X. Zhang, B. Yu, and X. Dong, "Power and discrete rate adaptation in BER constrained wireless powered communication networks," IET Communications, vol. 12, no. 18, pp. 2213-2221, 2018. 


$$
\begin{aligned}
& \mathcal{P}\left\{\log \left(1+S_{u 1 k_{1}}\right) \geq r \cap \log \left(1+S_{p d f, k_{2}}\right) \geq r\right\} \approx \int_{0}^{\frac{\alpha_{3}}{\alpha_{1}}} f\left(x_{0}\right)+\sum_{n=1}^{N} \frac{f^{(n)}\left(x_{0}\right)}{n !}\left(x_{1}-x_{0}\right)^{n} \mathrm{~d} x_{1} \\
& =\sum_{l=1}^{L} \int_{\gamma_{l}}^{\gamma_{l+1}} f\left(m_{l}\right)+\sum_{n=1}^{N} \frac{f^{(n)}\left(m_{l}\right)}{n !}\left(x_{1}-m_{l}\right)^{n} \mathrm{~d} x_{1}=\sum_{l=1}^{L}\left[f\left(m_{l}\right)\left(\gamma_{l+1}-\gamma_{l}\right)+\sum_{n=1}^{N} \frac{f^{(n)}\left(m_{l}\right)}{(n+1) !}\left(\left(\gamma_{l+1}-m_{l}\right)^{n+1}-\left(\gamma_{l}-m_{l}\right)^{n+1}\right)\right] .
\end{aligned}
$$

[4] H. Ju and R. Zhang, "Throughput maximization in wireless powered communication networks," IEEE Transactions on Wireless Communications, vol. 13, no. 1, pp. 418-428, January 2014.

[5] C. Guo, B. Liao, and L. Huang, "Time allocation and load balancing in multi-cell wireless powered communication networks," IEEE Access, vol. 4, pp. 7795-7805, 2016.

[6] J. Yang, J. Hu, K. Lv, Q. Yu, and K. Yang, "Multi-dimensional resource allocation for uplink throughput maximisation in integrated data and energy communication networks," IEEE Access, vol. 6, pp. 47163 47 180, 2018.

[7] Q. Wu, M. Tao, D. W. Kwan Ng, W. Chen, and R. Schober, "Energyefficient resource allocation for wireless powered communication networks," IEEE Transactions on Wireless Communications, vol. 15, no. 3, pp. 2312-2327, March 2016.

[8] S. Enayati, H. Saeedi, H. Pishro-Nik, and H. Yanikomeroglu, "Optimal altitude selection of aerial base stations to maximize coverage and energy harvesting probabilities: A stochastic geometry analysis," IEEE Transactions on Vehicular Technology, vol. 69, no. 1, pp. 1096-1100, 2020.

[9] M. Ku, W. Li, Y. Chen, and K. J. Ray Liu, "On energy harvesting gain and diversity analysis in cooperative communications," IEEE Journal on Selected Areas in Communications, vol. 33, no. 12, pp. 2641-2657, 2015.

[10] W. Saad, M. Bennis, and M. Chen, "A vision of $6 \mathrm{G}$ wireless systems: Applications, trends, technologies, and open research problems," IEEE Network, vol. 34, no. 3, pp. 134-142, 2020.

[11] C. Hu and Y. Dong, "Age of information of two-way data exchanging systems with power-splitting," Journal of Communications and Networks, vol. 21, no. 3, pp. 295-306, 2019.

[12] R. D. Yates and S. K. Kaul, "The age of information: Real-time status updating by multiple sources," IEEE Transactions on Information Theory, vol. 65, no. 3, pp. 1807-1827, 2019.

[13] A. Kosta, N. Pappas, and V. Angelakis, Age of Information: A New Concept, Metric, and Tool, 2017.

[14] A. Arafa, J. Yang, S. Ulukus, and H. V. Poor, "Age-minimal transmission for energy harvesting sensors with finite batteries: Online policies," IEEE Transactions on Information Theory, vol. 66, no. 1, pp. 534-556, 2020.

[15] B. T. Bacinoglu, Y. Sun, E. Uysal, and V. Mutlu, "Optimal status updating with a finite-battery energy harvesting source," Journal of Communications and Networks, vol. 21, no. 3, pp. 280-294, 2019.

[16] X. Zheng, S. Zhou, Z. Jiang, and Z. Niu, "Closed-form analysis of nonlinear age of information in status updates with an energy harvesting transmitter," IEEE Transactions on Wireless Communications, vol. 18, no. 8, pp. 4129-4142, 2019.

[17] X. Wu, J. Yang, and J. Wu, "Optimal status update for age of information minimization with an energy harvesting source," IEEE Transactions on Green Communications and Networking, vol. 2, no. 1, pp. 193-204, 2018.

[18] I. Krikidis, "Average age of information in wireless powered sensor networks," IEEE Wireless Communications Letters, vol. 8, no. 2, pp. 628-631, 2019.

[19] N. Hirosawa, H. Iimori, K. Ishibashi, and G. T. F. D. Abreu, "Minimizing age of information in energy harvesting wireless sensor networks," IEEE Access, vol. 8, pp. 219934-219945, 2020.

[20] S. Leng and A. Yener, "Age of information minimization for an energy harvesting cognitive radio," IEEE Transactions on Cognitive Communications and Networking, vol. 5, no. 2, pp. 427-439, 2019.

[21] M. A. Abd-Elmagid, H. S. Dhillon, and N. Pappas, "AoI-optimal joint sampling and updating for wireless powered communication systems," IEEE Transactions on Vehicular Technology, vol. 69, no. 11, pp. 14110 $14115,2020$.

[22] Y. Dong, P. Fan, and K. B. Letaief, "Energy harvesting powered sensing in IoT: Timeliness versus distortion," IEEE Internet of Things Journal, vol. 7, no. 11, pp. 10897-10911, 2020.
[23] Q. Gu, G. Wang, R. Fan, F. Li, H. Jiang, and Z. Zhong, "Optimal resource allocation for wireless powered sensors: A perspective from age of information," IEEE Communications Letters, vol. 24, no. 11, pp. 2559-2563, 2020.

[24] M. A. Abd-Elmagid, H. S. Dhillon, and N. Pappas, "A reinforcement learning framework for optimizing age of information in RFpowered communication systems," IEEE Transactions on Communications, vol. 68, no. 8, pp. 4747-4760, 2020.

[25] H. Ko, H. Lee, T. Kim, and S. Pack, "Information freshness-guaranteed and energy-efficient data generation control system in energy harvesting internet of things," IEEE Access, vol. 8, pp. 168 711-168720, 2020.

[26] H. Zheng, K. Xiong, P. Fan, Z. Zhong, and K. B. Letaief, "Age of information-based wireless powered communication networks with selfish charging nodes," IEEE Journal on Selected Areas in Communications, vol. 39, no. 5, pp. 1393-1411, 2021.

[27] Y. Dong, Z. Chen, and P. Fan, "Timely two-way data exchanging in unilaterally powered fog computing systems," IEEE Access, vol. 7, pp. 21 103-21 117, 2019.

[28] A. Arafa and S. Ulukus, "Age-minimal transmission in energy harvesting two-hop networks," in GLOBECOM 2017 - 2017 IEEE Global Communications Conference, 2017, pp. 1-6.

[29] — - "Timely updates in energy harvesting two-hop networks: Offline and online policies," IEEE Transactions on Wireless Communications, vol. 18, no. 8, pp. 4017-4030, 2019.

[30] Z. Zhou, C. Fu, C. J. Xue, and S. Han, "Energy-constrained data freshness optimization in self-powered networked embedded systems," IEEE Transactions on Computer-Aided Design of Integrated Circuits and Systems, vol. 39, no. 10, pp. 2293-2306, 2020.

[31] Y. Khorsandmanesh, M. J. Emadi, and I. Krikidis, "Average peak age of information analysis for wireless powered cooperative networks," IEEE Transactions on Cognitive Communications and Networking, pp. 1-1, 2021.

[32] T. D. P. Perera, D. N. K. Jayakody, I. Pitas, and S. Garg, "Age of information in swipt-enabled wireless communication system for 5GB," IEEE Wireless Communications, vol. 27, no. 5, pp. 162-167, 2020.

[33] Y. Zheng, Y. Zhang, Y. Wang, J. Hu, and K. Yang, "Create your own data and energy integrated communication network: A brief tutorial and a prototype system," China Communications, vol. 17, no. 9, pp. 193209, 2020.

[34] R. Karli, A. Bouchalkha, and K. Alhammadi, "Power consumption and battery life study of a two-node wireless sensor system," in 2016 th International Conference on Electronic Devices, Systems and Applications (ICEDSA), 2016, pp. 1-4.

[35] S. Luo, R. Zhang, and T. J. Lim, "Optimal save-then-transmit protocol for energy harvesting wireless transmitters," IEEE Transactions on Wireless Communications, vol. 12, no. 3, pp. 1196-1207, 2013.

[36] K. Lv, J. Hu, Q. Yu, and K. Yang, "Throughput maximization and fairness assurance in data and energy integrated communication networks," IEEE Internet of Things Journal, vol. 5, no. 2, pp. 636-644, April 2018.

[37] M. Oshaghi and M. j. Emadi, "Throughput maximization of a hybrid EHSWIPT relay system under temperature constraints," IEEE Transactions on Vehicular Technology, pp. 1-1, 2019.

[38] Y. Hu, Y. Zhu, M. C. Gursoy, and A. Schmeink, "SWIPT-Enabled relaying in IoT networks operating with finite blocklength codes," IEEE Journal on Selected Areas in Communications, vol. 37, no. 1, pp. 74-88, January 2019.

[39] M. Abedi, H. Masoumi, and M. J. Emadi, "Power splitting-based SWIPT systems with decoding cost," IEEE Wireless Communications Letters, vol. 8, no. 2, pp. 432-435, 2019.

[40] S. Boyd and L. Vandenberghe, Convex Optimization, 2009, pp. 466-475.

[41] E. K. P. Chong and S. H. Zak, An Introduction to Optimization (4th Edition), 2013. 


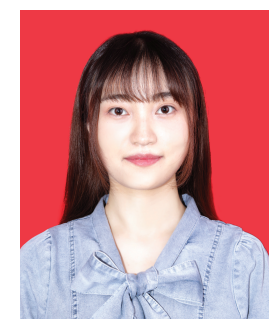

Yali Zheng is currently pursuing the Ph.D. degree in the School of Information and Communication Engineering, University of Electronic Science and Technology of China, Chengdu, China. Her current research interests include wireless power communication networks, data and energy integrated communication networks, resource allocation and age of information.

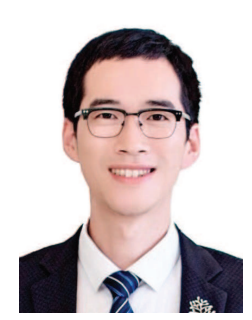

Jie Hu [S'11, M'16, SM'21] received his B.Eng. and M.Sc. degrees from Beijing University of Posts and Telecommunications, China, in 2008 and 2011, respectively, and received the Ph.D. degree from the School of Electronics and Computer Science, University of Southampton, U.K., in 2015. Since March 2016, he has been working with the School of Information and Communication Engineering, University of Electronic Science and Technology of China (UESTC), China. He is now a Research Professor. He has been elected into UESTC's Fundamental Research Program for Young Scientists since 2018. He also won UESTC's Academic Young Talent Award in 2019. Now he is supported by the "100 Talents" program of UESTC. His research now is mainly funded by National Natural Science Foundation of China (NSFC). He is an editor for both IEEE Wireless Communications Letters and IET Smart Cities. He serves for IEEE Communications Magazine, IEEE/CIC China Communications, Frontiers in Communications and Networks as well as ZTE communications as a guest editor. He is a program vice-chair for IEEE TrustCom 2020 and a technical program committee (TPC) chair for IEEE UCET 2021. He also serves as a TPC member for several prestigious IEEE conferences, such as IEEE Globecom/ICC/WCSP and etc. He has won the best paper award of IEEE SustainCom 2020. His current research focuses on wireless communications and resource management for $6 \mathrm{G}$, wireless information and power transfer as well as integrated communication, computing and sensing.

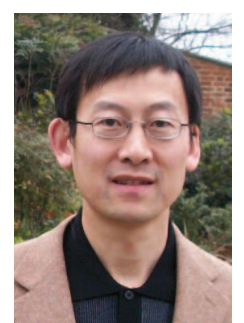

Kun Yang received his $\mathrm{PhD}$ from the Department of Electronic \& Electrical Engineering of University College London (UCL), UK. He is currently a Chair Professor in the School of Computer Science \& Electronic Engineering, University of Essex, leading the Network Convergence Laboratory (NCL), UK. $\mathrm{He}$ is also an affiliated professor at UESTC, China. Before joining in the University of Essex at 2003, he worked at UCL on several European Union (EU) research projects for several years. His main research interests include wireless networks and communications, IoT networking, data and energy integrated networks and mobile computing. He manages research projects funded by various sources such as UK EPSRC, EU FP7/H2020 and industries. He has published 300+ papers and filed 20 patents. He serves on the editorial boards of both IEEE (e.g., IEEE TNSE, IEEE ComMag, IEEE WCL) and non-IEEE journals. He is a Member of Academia Europaea (MAE). He is a Senior Member of IEEE (since 2008), a Fellow of IET and a Fellow of BCS. He is an IEEE ComSoc Distinguished Lecturer (2020-2021). 